\title{
Use of optical microscopy for evaluation of tooth structure
}

\author{
K. Banaszek a, J. Sawicki b, E. Wołowiec-Korecka b, ${ }^{*}$, J. Gorzędowski b, \\ D. Danowska-Klonowska c, J. Sokołowski a \\ a Chair of Restorative Dentistry, Department of General Dentistry, Medical University of Lodz, \\ ul. Pomorska 251, 92 - 213 Łódź, Poland \\ b Institute of Material Science and Engineering, Lodz University of Technology, \\ ul. Stefanowskiego 1/15, 90-924 Łódź, Poland \\ c Department of Cytophysiology, Histology and Embryology, Medical University of Lodz, \\ ul. Narutowicza 60, 91-036 Łódź, Poland \\ *Corresponding e-mail address: emilia.wolowiec@p.lodz.pl
}

\section{ABSTRAGT}

Purpose: There are many methods of examining tooth anatomy: some of them are applicable to the hard tissues, while others to the pulp and the root canal system. Every method has some advantages and disadvantages in terms of revealing specific anatomical or histological details of the tooth. The objective of the present work was to assess the use of macroscopic microscopy for evaluation of tooth anatomy and to compare the obtained images with those produced by the radiovisiographic method.

Design/methodology/approach: The study involved 4 upper canines extracted for periodontal reasons from patients of different age and sex. Initially, radiological images were taken in the vestibular-lingual and lateral projections by the radiovisiographic method using a KODAK 2000 system with a KODAK 6100 sensor. Subsequently, the specimens were ground with sandpaper and consecutive dental cross-sections were captured at approximately 0.05 $\mathrm{mm}$ increments using a NIKON AZ100 multizoom microscope.

Findings: The presented method, using side illumination and a digital capture device transferring the images to a computer, enables evaluation of tooth anatomy in terms of both shape and internal structure, and offers the dimensioning functionality.

Practical implications: Optical microscopy is a cheap method of examining the hard tissues of the tooth, including restorative materials, and for evaluating the development of primary and secondary dentin, as well as dentin affected by bacterial infection. A microscope used in conjunction with good software provides many opportunities for easy examination and dimensioning of particular anatomical features of the tooth on consecutive planes.

Originality/value: This is an honest methods review and analysis which are applied in modern prosthetics.

Keywords: Methodology of research; Optical microscopy; Anatomy and histology; Tooth; Transillumination

\section{Reference to this paper should be given in the following way:}

K. Banaszek, J. Sawicki, E. Wołowiec-Korecka, J. Gorzędowski, D. Danowska-Klonowska, J. Sokołowski, Use of optical microscopy for evaluation of tooth structure, Journal of Achievements in Materials and Manufacturing Engineering 79/1 (2016) 31-40.

\section{BIOMEDIGAL AND DENTAL ENGINEERING AND MATERIALS}




\section{Introduction}

The typical anatomical features of the crown and root(s) in the various types of teeth are commonly known.

There are many methods of studying tooth anatomy. Some are designed to examine the hard tissues by X-ray techniques [24], while others deal with the pulp [13] or the root canal system [20-23] Every method has some advantages and disadvantages in terms of revealing specific anatomical or histological details. Still other methods are used for examining the microarchitecture of dental tissues to determine the quality of adhesion between restorative materials and dental tissues. This paper presents a review of methods that may be applied for tooth examination, as well as proposes a new method.

\subsection{Scanning electron microscopy (SEM)}

One of the methods of imaging dental anatomy is scanning electron microscopy (SEM). As the tooth surface does not conduct electricity, it must be pre-coated with a conductive material (e.g., gold). For instance, in the first decade of the twentieth century this method was used to evaluate enamel and dentin degradation in an in vitro and in vivo study by Franklin Gordon et al. [5].

A scanning electron microscope has also been used by M. Camargo et al. [6] and Chu et al. [17] to examine dentin morphology with a view to determining the number of open and closed tubules per unit of area in human and bovine teeth. For the images to be taken, the specimens had to be appropriately prepared, that is, sectioned, ground, polished, sonicated, and coated with metal. This method was successfully used to show the location of tubules in the teeth and identify pathological areas with tubular sclerosis. The visualized tubules were from 1 to $5 \mu \mathrm{m}$ in diameter, which proves the good resolution of this method. While this method enabled analysis of surface morphology, it may not be used for differentiating structures in terms of their chemical composition due to the fact that the dentin must be pre-coated with a conductive material [6].

A special case of SEM use is environmental scanning electron microscopy (ESEM). Y. Wang et al. used this method to examine the structure of demineralized dentin in wet state to determine the depth of dentin demineralization as a result of etching with $35 \%$ phosphoric acid [8]. However, this method is characterized by low resolution and only allows for identifying the main structures, as well as distinguishing normal from demineralized dentin. It is not possible to reveal such details as collagen fibres or sclerotic plaques because of the low vacuum and interactions between electrons and the medium. On the other hand, the advantage of ESEM is the possibility to examine structures in situ, unmodified by preparatory work, drying, or vacuum conditions [8].

\subsection{Atomic force microscopy (AFM)}

Atomic force microscopy is a method that is essentially used to examine the topography of particular layers of biological specimens, typically following etching. It makes it possible to study not only the arrangement and porosity of a variety of structures, such as dentin, collagen fibres, and cementum, but also to determine their chemical composition thanks to the use of special probes. Sunita P. Ho et al. revealed the structure of etched dentin using a Nanoscope III Multimode AFM (DI-Veeco Instruments Inc., Santa Barbara) [2]. They determined the arrangement of collagen fibers, which act as a bridge between cementum and dentin. A major advantage of this method is that it enables resolutions of up to a few hundred nanometers (depending on the probe tip size) [2].

\subsection{Microcomputed tomography (micro-CT)}

For many years now, X-radiation has been successfully used to obtain images of teeth in situ as well as following their extraction, for research purposes. One of the increasingly widespread $\mathrm{X}$-ray-based methods is microcomputed tomography (micro-CT) as it can precisely reveal tooth structure and anatomy based on multiple plane projections. This method is usually characterized by a resolution revealing features larger than $10 \mu \mathrm{m}$ [1]. Sunita P. Ho et al. used microcomputed tomography (MicroXCT, Xradia Inc., Concord, CA) to examine the structure and orientation of collagen fibers, dentin, and cementum [2]. They employed special software to create a 3D representation of the various structures based on a series of 2D images [2].

Another example of micro-CT is the work of Amrit K. Rao et al., who compiled a detailed 3D atlas of human teeth using a large number of projections per plane (400 projections at $0.9^{\circ}$ increments) and a large number of planes (350 to 500 per tooth) to obtain high resolution [3]. The atlas presents not only the external outline of the tooth, but also the arrangement and structure of particular tissues 
forming the tooth. It should be emphasized that besides high-quality computed tomography equipment, the authors needed to use a computer with considerable computing power and with specialized software transforming projections into 3D images [3]. A similar study was conducted by Miloglu et al. to evaluate the root and canal configuration in humans [18].

Gin Chen and Yu-Chao Chang Yu et al. used computed tomography to evaluate apical resorption during orthodontic treatment [19].

Another example of the application of this nondestructive method is the work of B. Guillaume et al., which presents analysis of root canal morphology in wisdom teeth [4]. The authors acquired a large collection of planes consisting of 200 projections each (with a rotation of $0.9^{\circ}$ between consecutive projections in a given plane). Subsequently, they reconstructed 3D models using ANT software (Skyscan) in which enamel and dentin, as well as all calcifications and canal arrangement, could be easily distinguished. This method revealed tubules with a diameter of over $40 \mu \mathrm{m}$ [4].

\subsection{Raman microspectroscopy}

Raman microspectroscopy is a method characterized by high spatial resolution (1-1.5 $\mu \mathrm{m})$. It has been used to, e.g., obtain a detailed chemical map of demineralized dentin [8]. This method provides information primarily about the chemical composition of a layer rather than its topography. High-quality chemical maps of dentin may be very important in studying its different kinds, whose formation was affected by external factors, where no obvious visual or structural differences may be present [8].

\subsection{Harmonic generation microscopy}

Harmonic generation microscopy can be used to acquire high resolution (up to $1 \mu \mathrm{m}$ ) images. It is based on the phenomenon of harmonic generation in response to applied electromagnetic radiation (laser beam). For the examination of teeth, typically second- and third-harmonics are used, which are generated by dentin and canals (containing a liquid such as Hank's solution) in response to incident laser light. This enables exact imaging of planes and 3D model generation. In this method, the tooth surface is scanned point by point, collecting information on nonlinearly generated photons. This method has been used, e.g., to examine the architecture of dentin tubules and the distribution of collagen with an accuracy of $1 \mu \mathrm{m}$ [1].

\subsection{Electron probe microanalysis}

Electron probe microanalysis is a special case of electron microscopy, where the acquired image primarily contains information about the types of chemical substances present on the surface. It has been used, e.g., for examining the interactions between tooth structures and the adhesive materials employed to restore the affected tooth tissue [7]. This method easily distinguishes between, e.g., collagen fibres and hydroxyapatite crystals. The resolution is sufficient to determine substances occupying as little as $1 \mu \mathrm{m}$ [7].

\subsection{Digital photography}

A simple but effective method is imaging by means of a digital camera equipped with an appropriate macro lens. An example here is the study by B. Guillaume et al. [4], who used a computer-controlled photographic stand to photograph an immobilized tooth at a desired angle (e.g., a series of 72 images with a rotation of $5^{\circ}$ in a given plane). As many as 11 different angles of the tooth in respect of the horizontal plane were applied, resulting in a total of 792 images per tooth. In this way, 3D visualizations were created of the photographed teeth [4].

Another example is the use of an SLR camera (Canon EOS-300) with a $100 \mathrm{~mm}$ macro lens to photograph longitudinal tooth sections at $0.5 \mathrm{~mm}$ increments. The images were used to create a mathematical model describing the coronal contour. In this case, high resolution was not required, the important issue was to easily recognize the tooth contour on particular planes [9].

\subsection{Optical microscopy}

Optical microscopy may be a useful tool for examining general tooth anatomy and the basic features, such as the course and size of the root canal, the position and shape of the root apex, and the position of tooth tissues, enamel, dentin, and cementum. This method is simple thanks to uncomplicated preparation procedures (in contrast to, e.g., electron techniques), ease of microscope handling, and relatively low prices of equipment. Josue Martos et al. used this method to determine the position and shape of the root 
apex based on observations of 845 human teeth [10]. This method was also employed by Watson and Wilmot to analyse the influence of modern bonding agents on dentin smear layer thickness, tubular orientation, and immediate stress application on the dentin bonding agent [11].

\subsection{Confocal microscopy}

David B. Scott used a confocal optical microscope to examine tooth structure, including dentin, enamel thickness, cementum size, and canal morphology [12]. Such studies commonly employ dies, including fluorescent ones [1]. This method has also been used to determine the force of adhesion of systems binding composite materials to tooth tissues [14] and to evaluate the lipid composition of the organic matrix in mature enamel [15]. Another application example includes analysis of dentin structure and orientation as well as the processes occurring at the dentin-adhesive interface [16].

In the present work, we proposed an optical microscopy method based on macroscopic examination of transverse and longitudinal ground tooth sections. The objective of the paper was to evaluate the use of optical microscopy for examination of internal tooth anatomy and to compare the photographs obtained with the radiological images acquired by radiovisiography (RVG).

\section{Materials and methods}

The study material involved 4 upper canines extracted from patients of different age and sex. The extracted teeth were stored in formaldehyde.

\subsection{Radiovisiographic examination}

X-ray images were acquired in two projections (vestibular-lingual and lateral), prior to microscopic examination, using a KODAK 2000 system with a KODAK 6100 sensor. The length of the studied teeth was also measured.

\subsection{Tooth preparation}

For $24 \mathrm{~h}$ prior to examination, the teeth had been kept in $2.25 \%$ solution of sodium hypochlorite to remove any soft tissues. Subsequently, they were rinsed with distilled water and placed in an ultrasonic cleaner in $2.25 \%$ solution of sodium hypochlorite. Then, they were rinsed with distilled water again and dried with compressed air. Next, the teeth were embedded in epoxy resin in the horizontal position ( 2 teeth) and in the vertical position ( 2 teeth). The specimens were ground using a Metapolan 2 grinding machine with coarse sandpaper (180), and subsequently with increasingly fine sandpapers with grit size ranging from 240 to 800 . The embedded specimen was ground until the tooth was first revealed, at which time the specimen thickness was measured at a predetermined point with a Vernier scale. This specimen thickness was treated as a point of reference, and consecutive ground layers constituted the gradient of its thickness. The thickness of the ground layers amounted to an average of $0.05 \mathrm{~mm}$. Following every grinding procedure, the specimens were rinsed with distilled water and dried with compressed air. In the case of planes revealing the tooth chamber, the specimens were ultrasonicated for $5 \mathrm{~min}$ in 2.25 solution of sodium hypochlorite, and then in $15 \%$ sodium versenate. At the next step, the specimens were rinsed with distilled water, and finally dried with compressed air. Prior to each imaging step, specimen thickness was measured with a Vernier scale. Subsequent $0.05 \mathrm{~mm}$ layers were ground using grit 600 sandpaper. Images were acquired using a NIKON AZ100 multizoom microscope. Importantly, the specimens were lighted laterally, thanks to which the tooth was well illuminated, while the background was dark. In addition, the microscope made it possible to dimension the size of details in the images acquired.

\subsection{Optical examination in the macro region}

We used a Nikon AZ100 multizoom microscope with a $0.6 \times$ eyepiece and a magnification of $1 \times$ to $8 \times$. Side illumination and a digital capture device transferring the image to the computer were used.

\section{Result}

Results for two selected tooth specimens are presented below. One was ground in the vertical position (Figs. 1-4), and the other in the horizontal position (Figs. 5-7).

Fig. 1 shows photographs taken with a CANON 40D camera. Both images reveal a carious lesion on the vestibular surface of the coronal third of the root. Additionally, pathological tooth wear is visible on the incisal margin of the tooth (Fig. 1a). 


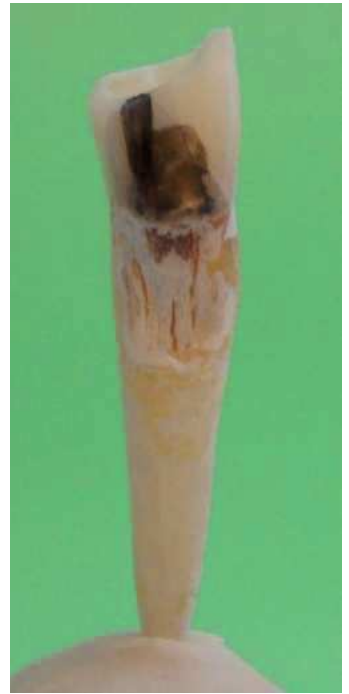

a)

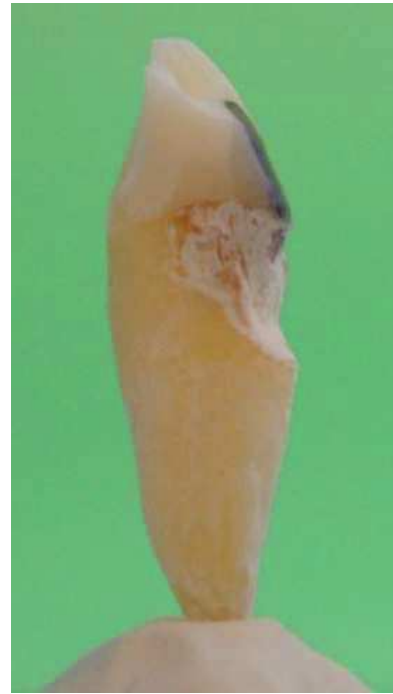

b)
Fig. 1. Macroscopic photograph of the specimen which was ground in the vertical position

Radiological examination by the radiovisiographic method (Fig. 2) showed in the vestibular-lingual projection (Fig. 2a) loss of the hard tissue of the tooth on the incisal margin of the crown, probably attributable to pathological attrition. Both projections (Fig. 2a, b) reveal tissue demineralization in the coronal and radical parts of the tooth indicating a carious lesion approximately one third of the width and reaching the pulp chamber (corresponding to approximately $30 \%$ of the volume of the tooth in question).

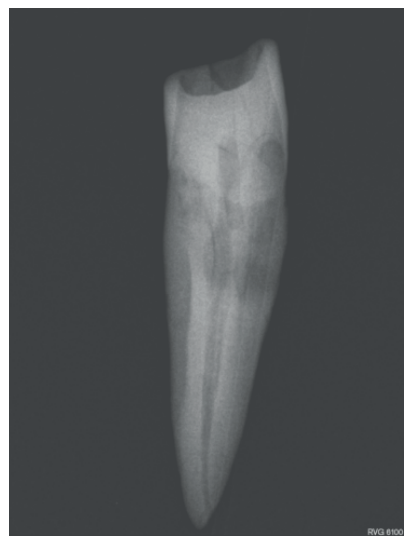

a)

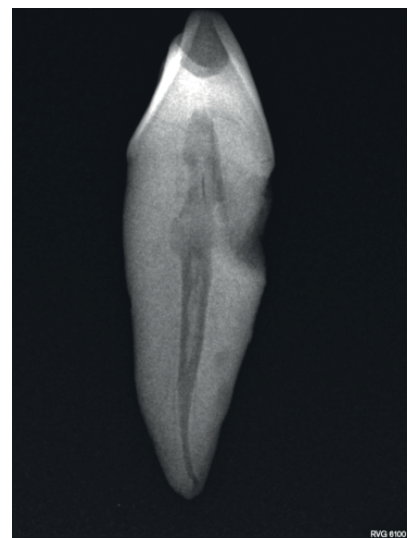

b)
Fig. 2. Radiographic images of the specimen which was ground in the vertical position: a) vestibular-lingual projection; b) lateral projection

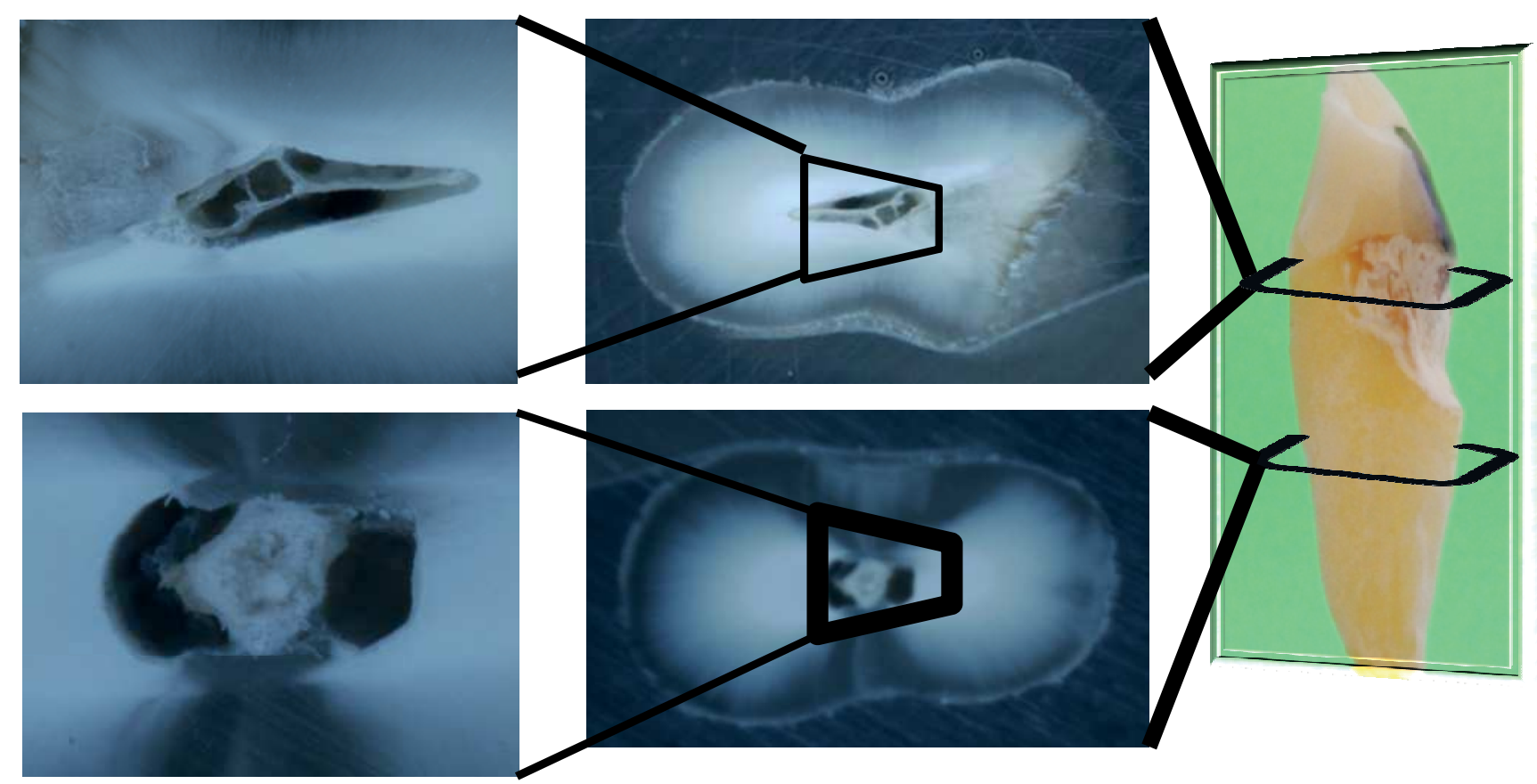

Fig. 3. Macroscopic photographs of the specimen which was ground in the vertical position acquired from an optical microscope 

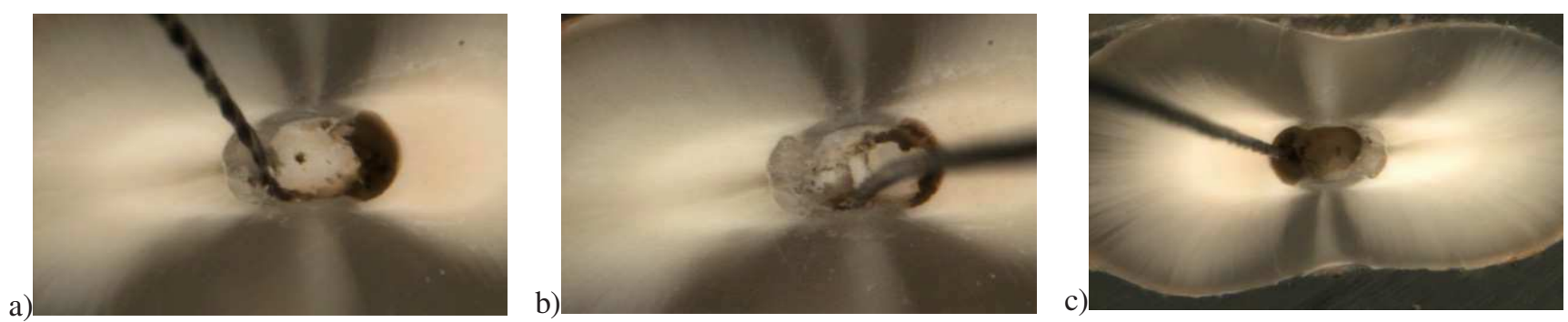

Fig. 4. Macroscopic photographs of the specimen which was ground in the vertical position showing an endodontic tool

The lateral projection reveals linear calcification in the coronal half of the root. Additionally, two small lucent spots in the apical third of the root can be seen (Fig. 2b).

The ground sections from two selected areas presented in Fig. 3 show a calcified, irregular structure in the lumen of the root canal (Fig. 3). The structure was hard and crumbled under the mechanical influence of an endodontic tool (Fig. 4).

a)

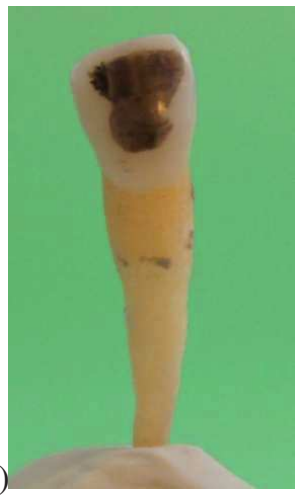

b)

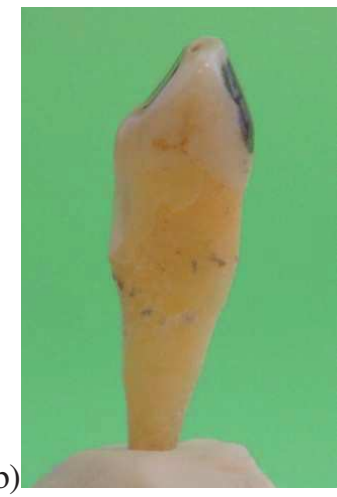

Fig. 5. Macroscopic photographs of the specimen ground in the horizontal position

Fig. 5 shows images of a canine from the vestibular side (Fig. 5a) and from the lateral side (Fig. 5b).
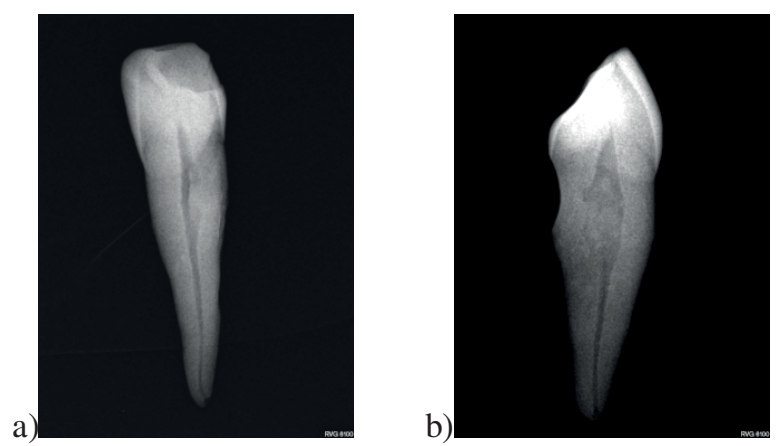

Fig .6. Radiographic images of the specimen which was ground in the horizontal position

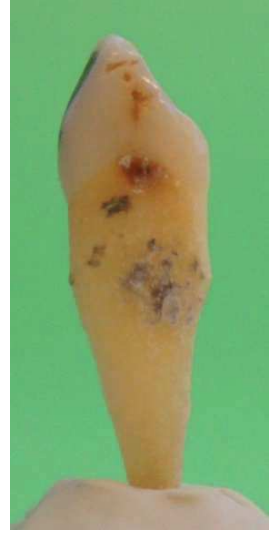

a)

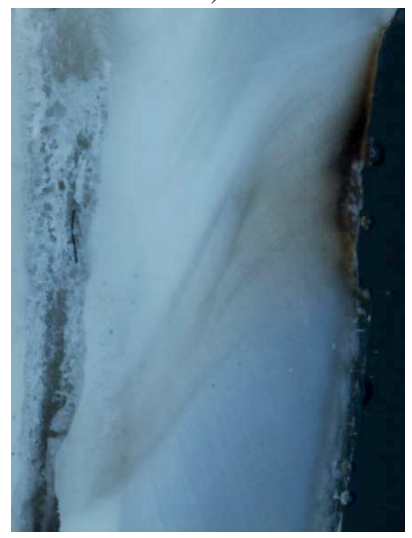

c)

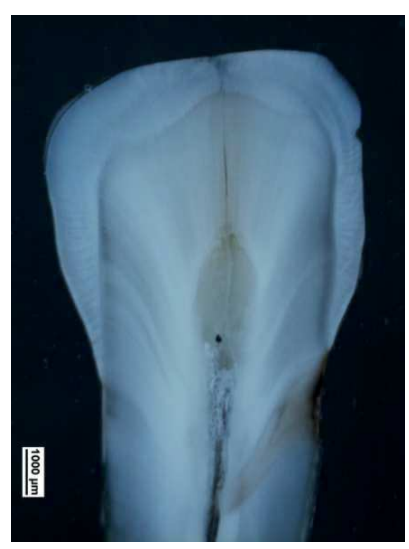

b)

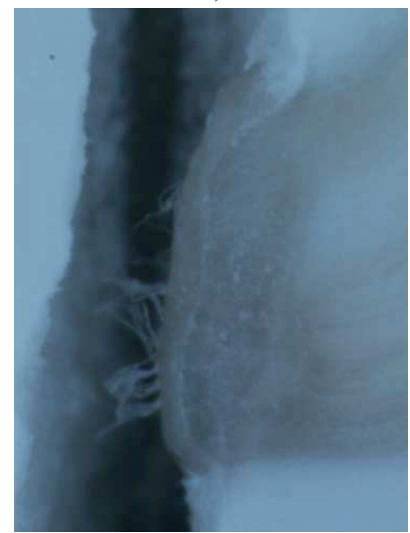

d)
Fig. 7. A macroscopic photograph and images acquired following horizontal grinding of the specimen

A radiovisiographic examination in the vestibularlingual projection (Fig. 6a) revealed demineralization of the hard tissue of the tooth on the incisal margin and the lateral side of the crown, which implies a class IV lesion according to Black's classification. In the anteroposterior dimension, one can see a loss of hard tissue on the incisal margin which probably arose due to attrition. The root tissue clearly exhibits a lucent area indicating the presence of caries (Fig. 6a), while Fig. 6b shows a cavity on the 
lingual side. A major opacification of the tissue is visible in the initial segment of the canal (in the lower part of the pulp chamber and the upper part of the root canal) (Fig. 6b).

Fig. 7 shows the extent of the pathological process. In the ground section, one can distinguish two types of pathological dentin under the carious focus. Immediately near the carious lesion, there arose a so-called dead space. This process occurs when a group of closely clustered odontoblasts becomes very intensively irritated, which leads to increased deposition of hydroxyapatite crystals in dentin tubules. As a result, the odontoblast processes die off and the lumen of the tubules becomes partially obstructed. Therefore, those parts of dentin which are immediately adjacent to such tubules are deprived of nutrients. In optical microscopic examination of the tooth ground section, the dead space is represented by dark strands as light is refracted by the air present in the dentin tubules deprived of cytoplasmic processes.

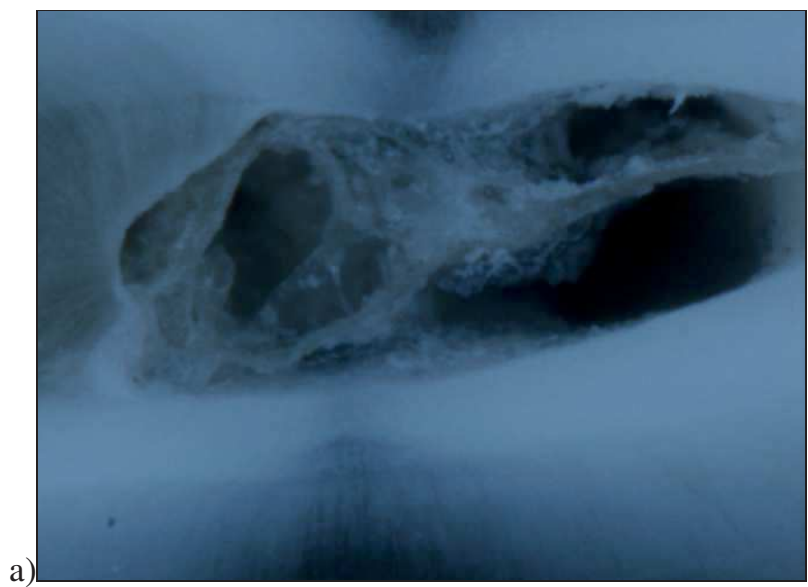

b)

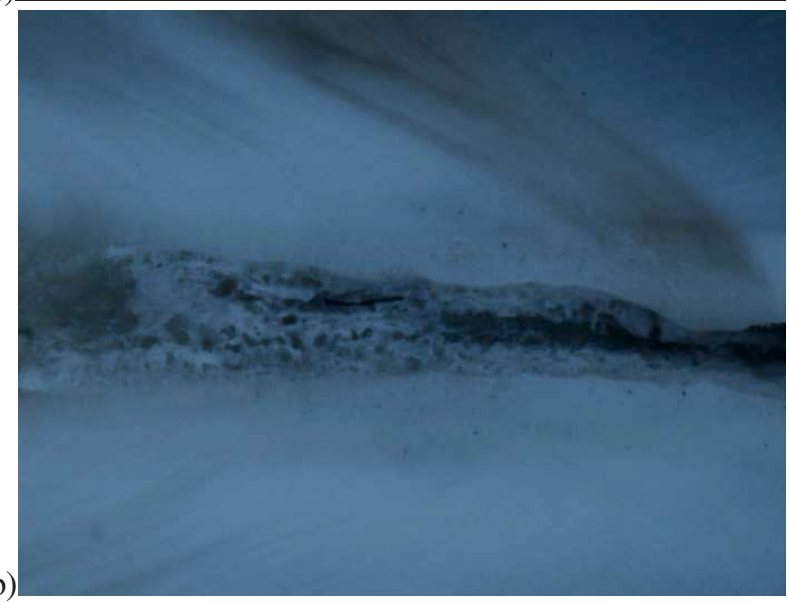

Fig. 8. Macroscopic images acquired using an optical microscope showing a) a transverse ground section and b) a longitudinal ground section
Another type of pathological change to dentin involves reactionary dentin, deposited immediately beneath dead tooth tissue, which is pathological secondary dentin. It is formed as a result of a defensive reaction of dentin in response to, e.g., carious lesions. In this case, odontoblasts produce dentin at a much faster rate, but only in the area affected by the pathological process. The orientation of the tubules is irregular, and they are not continuous with the physiological secondary dentine tubules. As can be seen in the section, this leads to local changes in the root canal contour.

Fig. 8 shows cross-sections of a pulp chamber which exhibits secondary physiological dentin. As can be seen, it occupies a large part of the chamber. This kind of dentin is formed throughout the lifetime of the tooth, as it is stimulated by physiological mastication-related stimuli. This process leads to decreased chamber and root canal lumen. Secondary physiological dentin is visible as a thin, uneven layer deposited on primary dentin. This type of dentin is characterized by a smaller number of tubules than primary dentin, and its structure is much less ordered.

\section{Discussion}

Examination of tooth anatomy by means of an optical microscope was found to be very useful, as high-resolution high-contrast images were acquired, revealing the minutest features, including interfaces between the various tooth tissues, some of which are only a dozen micrometers or so in size. Furthermore, the anatomical details present in the analysed images may be dimensioned. The X-ray method is very helpful in evaluation of relatively large elements of tooth structure, but it may not be sufficiently exact for examining the shape of root canals including accessory canals and their minute features (of an order of hundreds of micrometers). In dental practice, X-rays are the most frequent form of imaging during treatment, but they show only one projection of the entire tooth, which is a very general representation and may be erroneously interpreted due to overlapping layers. This may be avoided by the use of computed tomography. However, such an examination, affording high quality 3D images, implies a significant increase in costs as well as a greater dose of radiation and longer scanning time. In order to obtain specimens revealing the morphology of the root canal system, it would be necessary to apply a contrast agent, which is practically impossible in in vivo settings, and which is also not easy in extracted teeth. Nevertheless, X-rays and radiovisiography continue to be the most popular techniques for tooth imaging immediately preceding root canal treatment. They 
provide some basic information as to the morphology of the canal that is used for treatment or in vitro studies. However, these techniques were found by us insufficient for detailed analysis of root anatomy.

The method employing a photographic camera and macro lenses was proposed as an alternative that requires simpler equipment for in vitro studies than that necessary for specialist microscopy. Furthermore, a photographic frame is larger, which makes it possible to record the entire area of the largest longitudinal sections in one photograph. Initially, it seemed that this method would meet the researchers' expectations as to the quality and discernibility of details (the main root canal was very well visible). However, we found that in comparison to images acquired with an optical microscope, macro photographs do not exhibit sufficient contrast or magnification to distinguish particular tooth tissues. Moreover, dimensioning such photographs is more difficult, as it is necessary to take a preliminary photograph and a previously prepared scale and to place this scale in the photographs. Such a setup does not enable the flexible and quick dimensioning capacity along any axis that is offered by an optical microscope (Fig. 9). Another argument for optical microscopy is greater image reproducibility than that in macro photography, where the light and lamp settings as well as the specimen angle are more prone to change between series.

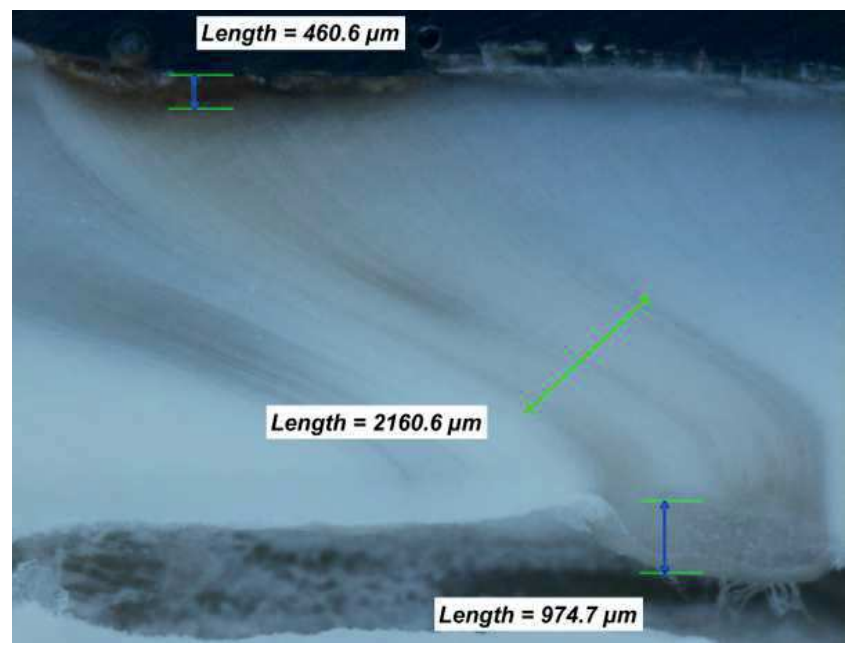

Fig. 9. Sample dimensioning of the analysed specimens

The presented optical microscopy method was used to compare two specimens - one examined in the vertical position (transverse ground section) and the other in the horizontal position (longitudinal ground section). This method provides exact information about the position and morphology of the canal and hard tissues of the tooth. Moreover, the repeatability of parameters in the presented method is easily achievable. The acquired images revealed such anatomical details as enamel and dentin thickness and the extent of the pathological process. It was also possible to trace the course of the canal. The study methodology of removing the smear layer was found to be useful for revealing minute features of the many layers of the analysed specimens by means of a Nikon AZ100 multizoom microscope. Similar specimen preparation procedures were employed by Chen and Chang for removing dentin debris and the smear layer during rotary root-canal instrumentation [19].

The methods reviewed above in the introduction offer very precise information (with details of $1 \mu \mathrm{m}$ and less) about the topography of the surface, as well as its morphology and chemical composition. They are very useful in determining the various structures present in the tooth. However, it should be pointed out that some of them are excessively exact for the purposes of defining general tooth anatomy. Apart from image quality, another important factor is the cost of imaging instruments. To give a general idea, the approximate prices of the equipment necessary to perform the reviewed procedures are listed below: a microCT scanner - $\$ 200,000$ to $\$ 400,000$; a scanning electron microscope (SEM) - \$50,000 to $\$ 200,000$; a Raman microscope - at least $\$ 30,000$; an atomic force microscope (AFM) - at least $\$ 20,000$. In contrast, the cost of an optical microscope which may be used for examination of tooth anatomy is significantly lower.

Thus, given the economic factor, relatively simple preparatory procedures, and easy microscope handling, optical microscopy seems to be a very attractive method for examination of tooth anatomy, both in terms quality and cost. The acquired images may be processed to obtain three-dimensional models or subjected to computer analysis. The present study shows that the best results of tooth anatomy evaluation are obtained using an optical microscope for examination of teeth embedded in epoxy resin. In this study approach, of fundamental importance is appropriate specimen preparation. A microscope in conjunction with good software gives many opportunities for easy observation and dimensioning of particular anatomical features of the tooth on a series of planes.

Our study has shown that optical microscopy can be used for inexpensive examination of tooth structure and morphology. This method can be applied for observation of the hard tissues of the tooth with restorative materials as well as for evaluation of the development of primary and secondary dentin, including dentin affected by bacterial infection. 


\section{Conclusions}

Optical microscopy is a cheap method of examining the hard tissues of the tooth, including restorative materials, and for evaluating the development of primary and secondary dentin, as well as dentin affected by bacterial infection. A microscope used in conjunction with good software provides many opportunities for easy examination and dimensioning of particular anatomical features of the tooth on consecutive planes.

\section{References}

[1] R. Elbaum, E. Tal, A.I. Perets et al., Dentin microarchitecture using harmonic generation microscopy, Journal of Dentistry 35 (2007) 150-155.

[2] P.H. Sunita, B. Yu, Y. Wenbing et al., Structure, chemical composition and mechanical properties of human and rat cementum and its interface with root dentin, Acta Biomaterialia 5 (2009) 707-718.

[3] A.K. Rao, K. Montgomery, W.P. Brown, E. Herbranson, 3-D interactive atlas of human tooth anatom, International Congress Series 1256 (2003) 93-98.

[4] B. Guillaume, J.P. Lacoste, N. Gaborit et al., Microcomputed tomography used in the analysis of the morphology of root canals in extracted wisdom teeth, British Journal of Oral and Maxillofacial Surgery 44 (2006) 240-244.

[5] F. Garcia-Godoy, N. Krämer, A. Feilzer, R. Frankenberger, Long-term degradation of enamel and dentin bonds: 6-year results in vitro vs. in vivo, Dental Materials 26 (2010) 1113-1118.

[6] M.A. Camargo, M.M. Marques, A.A. de Cara, Morphological analysis of human and bovine dentine by scanning electron microscope investigation, Archives of Oral Biology 53 (2008) 105-108.

[7] M. Hotta, Y. Li, I. Sekine, Mineralization in bovine dentin adjacent to glass-ionomer restorations. Journal of Dentistry 29 (2001) 211-215.

[8] Y. Wang, X. Yao, Morphological/chemical imaging of demineralized dentin layer in its natural, wet state, Dental Materials 26 (2010) 433-442.

[9] H. Yu, Q. Li, J. Hu, Y. Wang, An improved method to analyse tooth and restoration contour using image analysis: Application in the maxillary anterior teeth in Chinese population, Archives of Oral Biology 53 (2008) 503-508.

[10] J. Martos, C. Lubian, F.M. Luiz, L.F.M. Silveira, Suita L.A. de Castro, C.M. Ferrer Luque, Morphologic Analysis of the Root Apex in Human Teeth, Journal of Endodontics 36 (2010) 664-667.
[11] T.F. Watson, D.M. de J. Wilmot, A confocal microscopic evaluation of the interface between Syntac adhesive and tooth tissue, Journal of Dentistry 20 (1992) 302-310.

[12] D.B. Scott, Microscopic studies of dental tissues, II. Optical microscopy of tooth surfaces, Oral Surgery, Oral Medicine, Oral Pathology 5 (1952) 638-645.

[13] H.M. Alwas-Danowska, M.C.D.N.J.M. Huysmans, E.H. Verdonschot, Effects of alternating and direct electrical current application on the odontoblastic layer in human teeth: an in vitro study, International Endodontic Journal 32 (1999) 459-463.

[14] S. Sauro, T.F. Watson, I. Thompson, A. Banerjee, Onebottle self-etching adhesives applied to dentine airabraded using bioactive glasses containing polyacrylic acid: An in vitro microtensile bond strength and confocal microscopy study, Journal of Dentistry 40 (2012) 896-905.

[15] G. Veerappan, S. Hsu Chin-Ying, Characterization of lipid in mature enamel using confocal laser scanning microscopy, Journal of Dentistry 31 (2003) 303-311.

[16] B.M. Griffiths, T.F. Watson, M. Sherriff, The influence of dentine bonding systems and their handling characteristics on the morphology and micropermeability of the dentine adhesive interface, Journal of Dentistry 27 (1999) 63-71.

[17] C. Chia-Yi, K. Tien-Chun, C. Shu-Fang, S. Yow-Chyun, L. Chun-Pin, Comparison of the microstructure of crown and root dentin by a scanning electron microscopic study, Journal of Dental Sciences 5 (2010) 14-20.

[18] O. Miloglu, H. Arslan, C. Barutcigil, K. Cantekin, Evaluating root and canal configuration of mandibular first molars with cone beam computed tomography in a Turkish population, Journal of Dental Sciences 8 (2013) 80-86.

[19] C. Gin, C. Yu-Chao, Effects of liquid- and paste-type EDTA on smear-layer removal during rotary rootcanal instrumentation, Journal of Dental Sciences 6 (2011) 41-47.

[20] F.J. Vertucci, Root canal anatomy of the human permanent teeth, Oral Surgery, Oral Medicine, Oral Pathology, Oral Radiology, and Endodontology 58 (1984) 589-599.

[21] B.P.F.A Gomes, H.H. Rodrigues, N. Tancredo, The use of a modeling technique to investigate the root canal morphology of mandibular incisors, International Endodontic Journal 29 (1996) 29-36.

[22] M. Miyashita, E. Kasahara, E. Yasuda, A. Yamamoto, T. Sekizawa, Root canal system of the mandibular incisor, Journal of Endodontics 23 (1997) 479-483. 
[23] N. Kartal, Yanikoglu F.C. Root canal morphology of mandibular incisiors, Journal of Endodontics 18 (1992) 562-564.
[24] F. Pineda, Y. Kutter, Mesiodistal and buccolingual roentgenographic investigation of 7.275 root canals, Oral Surgery 33 (1972) 101-110. 